\title{
IGUALDAD Y TRABAJO PASTORAL: \\ LA EXPERIENCIA DE LAS MUJERES \\ EN LOS MINISTERIOS RELIGIOSOS ORDENADOS \\ Y CONSAGRADOS EN MÉXICO
}

\author{
Sandra Villalobos Nájera
}

Universidad Nacional Autónoma de México

\section{RESUMEN}

Este trabajo es parte de una investigación cuyo objetivo es explorar la construcción de la igualdad como un derecho y las formas de participación de las mujeres dentro del campo religioso, a partir de la experiencia de religiosas católicas consagradas y pastoras de denominación bautista, luterana, metodista, presbiteriana y ecuménica. La investigación busca, a través de sus trayectorias pastorales, visibilizar su condición y situación dentro de las estructuras religiosas a las que pertenecen. El trabajo religioso y pastoral es considerado un aspecto fundamental del análisis, puesto que se trata de un eje articulador de experiencias relacionales, económicas y sociales en las que la desigualdad de género y las relaciones de poder implícitas y explícitas que lo organizan son más evidentes.

\section{PALABRAS CLAVE}

Igualdad, Trabajo pastoral, Reivindicaciones de justicia, Fuerza laboral complementaria

\section{ABSTRACT}

This work is part of a research paper whose objective is to explore the construction of equality as a right and the ways in which women participate in the religious field. Based on the pastoral experience of Consecrated Catholic women and Ordained Baptist, Lutheran, Methodist, Presbyterian, and Ecumenical women pastors, the research aims to make their condition and situation within their religious structures visible. Religious and pastoral work are considered key aspects in the analysis because they interconnect relational, economic and social experiences in which gender inequality and 
implicit and explicit power relations are more evident.

KEY WORDS

Equality, Pastoral work, Claims for justice, Supplementary workforce 


\section{INTRODUCCIÓN}

La historia del cristianismo, la conformación de sus comunidades y el mantenimiento de sus estructuras no podrían entenderse sin la participación de las mujeres. Las mujeres han participado en la historia, las narraciones, la vida cotidiana y en cada una de sus expresiones dentro de las diferentes iglesias; representan, incluso, el mayor número de asistentes a los servicios religiosos y la población que más tareas de cuidados y servicios proporciona a estas instituciones.

Sin embargo, no porque su asistencia y participación sea numerosa se puede considerar que se trata del reconocimiento o ejercicio de una igualdad manifestada dentro del campo religioso; por el contrario, no se debe confundir está abundante participación como un ejercicio de derechos ganados al interior de dicho campo, pues resulta fundamental revisar el tipo de posiciones que las mujeres ocupan, las responsabilidades que les son dadas, las sujeciones de las que son objeto y, por supuesto, las relaciones de poder en las que están inmersas dentro del mismo. El conjunto de lo anterior ofrece un panorama en el que resulta imposible negar la profunda desigualdad que se oculta bajo argumentos de Tradición religiosa e incluso de carácter que pretende ser divino, para mantener la sumisión y opresión de las mujeres a través de mecanismos como el bajo o nulo reconocimiento, la negación de su agencia y la omisión de sus derechos humanos fundamentales; formas, todas ellas, de mantener la organización y perpetuidad del campo religioso y sus estructuras.

En muchos casos, la participación de las mujeres se limita a actividades que reproducen, reafirman y mantienen la extensión de sus roles de género dentro del espacio doméstico con actividades de servicio y administración del cuidado y los recursos. La participación, sin lugar a dudas importante, ha quedado fuera del reconocimiento oficial, y por tanto público, dentro de la mayoría de las instituciones religiosas. Y aunque algunas denominaciones cristianas reconocen, dentro de su organización, la participación de las mujeres en puestos de liderazgo como en el caso de algunos ministerios religiosos, aún no se puede hablar de una situación de igualdad.

En México existen numerosos estudios sobre las diversas manifestaciones del fenómeno religioso, algunos de tipo teórico y metodológico desde diferentes campos disciplinarios, y muchos otros de carácter etnográfico, que pretenden dar cuenta de experiencias particulares acerca de la amplia gama de manifestaciones religiosas que existen y que se van conformando a partir del movimiento de sus actores.

En el caso de la experiencia de las mujeres de denominación cristiana, los estudios realizados en México tienden más a la investigación de los movimientos católicos en torno al derecho a decidir y de los grupos pentecostales y neopentecostales y de sus nuevas configuraciones religiosas.

De manera más específica, dentro de aquellos en los que la experiencia de las mujeres es el punto de partida, se puede mencionar la importancia de las investigaciones: de Elizabeth Juárez (2000) sobre la reproducción de roles tradicionales de género; de Patricia Fortuny (2001) sobre la representación y la figura femenina; de Alberto Velázquez (2013) sobre la institucionalización religiosa del género; 
$y$, de manera destacada, la investigación de De la Torre y Fortuny (1991) sobre participación y representaciones simbólicas en mujeres de la Luz del Mundo.

En países como Brasil, donde la experiencia de investigación sobre el fenómeno religioso resulta muy amplia tanto por la gran diversidad religiosa que lo caracteriza como por la abundante producción teológica, los estudios sobre la participación de las mujeres cristianas tanto en el ámbito teológico y eclesial como en el ámbito político, nos muestran una participación que se torna más visible en la esfera pública, o bien, más agencial dentro del mismo campo.

Tal es el caso de los estudios de Campos Machado (2005) y Campos Machado et al. (2006) sobre relaciones de género en grupos pentecostales y participación política vinculados con el trabajo de las mujeres provenientes de grupos evangélicos en el ámbito legislativo en Brasil; del trabajo de Rosane Aparecida De Souza (2015) sobre mujeres que fundan nuevas Iglesias, y de Claudirene Bandini (2015) sobre la experiencia de mujeres en las misiones de Asamblea de Dios. Todos los anteriores colocan el acento en nuevas formas de participación en las que intervienen las mujeres desde su identidad religiosa.

Estos trabajos contribuyen a entender el tipo de participación que las mujeres tienen dentro del campo religioso y los diferentes movimientos generados por ellas mismas, todos desde una realidad latinoamericana similar, en contraparte con otros contextos, como los europeos, donde las mujeres han participado de manera más activa dentro del ámbito teológico y eclesial y en donde, por dar un ejemplo, la ordenación de las mujeres ha sido una realidad desde hace décadas y temas como la ordenación de hombres y mujeres con orientaciones sexuales diferentes a la heterosexual o el celibato de los sacerdotes, son discutidos dentro de las mismas instituciones religiosas.

\section{LAS MUJERES Y EL CAMPO RELIGIOSO}

Para hablar de igualdad dentro del campo religioso, parto en este artículo de la perspectiva bourdiana del campo como una red o configuración de relaciones objetivas entre posiciones, las cuales están definidas objetivamente en la distribución del poder y cuya relación de fuerzas es lo que define la estructura del campo (Bourdieu y Wacquant, 2005: 150-152).

Este campo de juego en el que existe una relación de fuerzas, una configuración de relaciones entre sus agentes y la disputa del capital, es donde se puede ubicar a las mujeres dentro de lo que Bourdieu (2006) llama los laicos, es decir, aquellos agentes desposeídos de los bienes de salvación que se encuentran a cargo de los especialistas religiosos de la producción y reproducción de los saberes dentro del campo.

[...] la constitución de un campo religioso es correlativa de la desposesión objetiva de los que están excluidos de él y que se encuentran constituidos por eso mismo en tanto que laicos (o profanos en el doble sentido del término) desposeídos del capital religioso (como trabajo simbólicamente acumulado) y que reconocen la legitimidad de esta desposesión por el sólo hecho de que las desconocen como tal (Bourdieu, 2006: 43).

Las mujeres no han sido consideradas parte de este cuerpo de especialistas al interior de la mayoría de las Iglesias de tradición cristiana; su papel dentro 
de las instituciones religiosas ha estado mayormente destinado a labores relacionadas con la extensión de sus roles de género, como la enseñanza religiosa a niños y niñas, al cuidado de enfermos y desvalidos, a la organización de eventos para recaudar recursos económicos (no así para decidir sobre los mismos), a la administración de dispensarios u otros deberes o ministerios, como en los casos de los ministerios de canto y danza, entre otras. La producción teológica y la participación y responsabilidades en puestos de liderazgo queda, por lo general, fuera de las primeras opciones de participación para las mujeres dentro de estas instituciones.

Al respecto, Juan José Tamayo menciona que en estas instituciones, a las que él llama "patriarquías" dado que su organización se configura a nivel patriarcal, las mujeres dentro de las religiones no han sido consideradas sujetos morales, pues requieren de guías para distinguir el bien y el mal; tampoco han sido consideradas sujetos religiosos, dado que la divinidad es masculina y por lo tanto sólo puede ser representada por un varón; ni sujetos teológicos, puesto que se trata de un terreno que no les corresponde (Tamayo, 2011: 213-215). Tres aspectos fundamentales que contribuyen a que la participación de las mujeres se dé desde la periferia, puesto que, al no ser consideradas como agentes en la producción de saberes y prácticas y cuya conciencia es capaz de discernir de manera autónoma, tanto sus decisiones como sus acciones quedan fuera de los espacios de injerencia institucional dentro de las estructuras eclesiales.

De esta manera se puede encontrar coincidencia entre ambas perspectivas. Las mujeres dentro del campo religioso no pertenecen al grupo de especialistas que poseen los bienes de salvación ni el capital religioso: desde la perspectiva bourdiana podemos ubicarlas como parte de los laicos desposeídos del capital, mientras que desde la postura de Tamayo esto podría explicarse debido a que no son consideradas sujetos morales, religiosos y teológicos dentro del campo.

Las religiones legitiman de múltiples formas la exclusión de las mujeres de la esfera pública, de la vida política, de la actividad intelectual y del campo científico, y limitan sus funciones al ámbito doméstico, a la esfera de lo privado, a la educación de los hijos e hijas, a la atención al marido, al cuidado de enfermos o personas mayores, etcétera. Cualquier tipo de actividad política o social se considera ajeno a la identidad femenina y un abandono de su verdadero ámbito de trabajo, que es el hogar, con la consiguiente culpabilización (Tamayo, 2011: 215).

Por todo lo anterior, el ejercicio de la igualdad como un derecho dentro del campo religioso se convierte en el punto de partida. Este espacio que para algunos es sólo un ámbito de la vida privada de las personas, es también uno de los lugares en el que las desigualdades, producto de la condición de género, se hacen más evidentes, sobre todo si consideramos que una parte de la estructura organizacional de las instituciones religiosas ha sido cimentada en función de la jerarquización y división de los sujetos que participan en su interior, como un criterio de pertenencia y exclusión, y también como elementos que determinan el estatus, las funciones y el tipo de participación que cada individuo tiene dentro de ese campo.

Este texto forma parte de una investigación sobre la manera como las mujeres construyen la igualdad como un derecho dentro del campo religioso y sus formas de participación a través del 
ejercicio de sus ministerios religiosos ordenados o consagrados. Se entiende por igualdad el ejercicio de derechos y obligaciones de las mujeres como sujetos políticos dentro de un contrato social (Lamas, 2001: 13), en que se reconocen y/o asumen como ciudadanas con oportunidades sociales, económicas y de participación política, en la mayoría de los casos fuera de sus espacios religiosos, pero también dentro de ellos. Y esto, aunque parezca contradictorio, destaca aún más el valor que esta construcción de igualdad tiene dentro de un campo religioso que por sí mismo no las dota de recursos, reconocimiento y representación como lo hace para otros actores dentro del mismo campo.

\section{ASPECTOS METODOLÓGICOS}

Se trata de una investigación cualitativa de tipo exploratorio cuyos ejes principales de análisis son la trayectoria de vida religiosa, los tipos de participación, el trabajo religioso y el ejercicio del poder en pastoras de denominación bautista, luterana, metodista, presbiteriana y ecuménica y en religiosas católicas consagradas, cuyos ministerios se destacan por una práctica religiosa de mayor autonomía, reconocimiento e incluso liderazgo al interior de sus comunidades de fe.

La investigación se desarrolla desde una epistemología y metodología feminista, cuyo centro es la experiencia de las mujeres cristianas dentro del ámbito religioso respecto al ejercicio de algunos de sus derechos y la forma como participan al interior de sus Iglesias considerando "distintos emplazamientos de las mujeres como sujetos cognoscibles y cognoscentes que se conocen y reconocen mutuamente" (Castañeda, 2008: 81), lo que contribuye a su reconocimiento como agentes sociales y políticos.

Como técnicas de recolección de datos se utilizaron métodos etnográficos y biográficos como la observación participante y el relato de vida. Respecto a la primera, la observación se llevó a cabo desde la postura de observador como participante, ${ }^{1}$ lo que permitió conocer y comprender el contexto, así como las actividades e interacciones entre las y los miembros de las comunidades religiosas a las que las participantes pertenecen, con el conocimiento de cada una y de cada uno de las y los involucrados. Las observaciones se realizaron en la asistencia a cultos religiosos, reuniones ecuménicas y principalmente en la asistencia a dos encuentros de mujeres investigadoras, teólogas y activistas que tuvieron lugar en la Ciudad de México en 2014 y 2015.

En cuanto al método biográfico, se seleccionó el relato de vida ${ }^{2}$ como una forma de obtener información sobre la trayectoria religiosa y pastoral de las participantes, puesto que es un método que permite captar relaciones y procesos.

Para este estudio se realizaron en total 16 entrevistas a profundidad en la Ciudad de México, ocho de ellas a mujeres pastoras de denominación bautista, luterana, metodista, presbiteriana y una autodenominada ecuménica y a tres religiosas católicas consagradas pertenecientes a las Congregaciones Filipense, Scalabriniana y de Santa

$1 \mathrm{La}$ postura de observador como participante permite, a quien investiga, participar en las actividades mientras el grupo es consciente de la observación de sus actividades (Kawulich, 2005).

2 La perspectiva etnosociológica contempla la existencia de categorías de situación, es decir, categorías que son comunes a todos los sujetos pero que se vuelven sociales cuando se perciben a través de la colectividad (Bertaux, 2005). 
Teresa del Niño Jesús, todas ellas de credo cristiano.

Lo anterior es importante dado que en un país cuya hegemonía religiosa es católica, se tiende a pensar que el equilibrio estaría en equiparar el número de mujeres católicas entrevistadas con el número de mujeres de Iglesias cristianas históricas también entrevistadas. Por este motivo, se hace la siguiente observación: la diferencia entre las mujeres entrevistadas no radica en el credo, puesto que todas son cristianas ${ }^{3}$ y con ello comparten una serie de elementos religiosos comunes, pero no así estructurales, pues pertenecen a diferentes instituciones eclesiales. La diferencia entonces, según la clasificación del Instituto Nacional de Estadística, Geografía e Informática (INEGI, 2015), se encuentra en la denominación a la que pertenecen, siendo esta última diferente en todas ellas.

Para fines de esta investigación, la diferencia entre las mujeres participantes radica, pues, en la denominación, clasificación que refleja las diferencias estructurales de sus instituciones eclesiales, y en que para las mujeres que pertenecen a la denominación católica no existe ninguna posibilidad de acceder a la ordenación pastoral de acuerdo a sus dogmas y normatividades institucionales, por lo que sólo pueden ejercer un ministerio legitimado a través de la misión o la consagración.

En el caso de las Iglesias cristianas históricas, la ordenación pastoral no es un hecho consolidado

3 Dentro del credo cristiano, en el documento de Clasificación de Religiones 2010 (INEGI, c2015), se puede encontrar a la Iglesia católica, la Iglesia ortodoxa, las protestantes históricas o reformadas, las pentecostales/evangélicas/ cristianas y las bíblicas diferentes de evangélicas. Como cristianismo se entiende el sistema de creencias y valores basadas en la doctrina de Jesucristo, como hijo de Dios, y la Biblia, como palabra divina. para todas las iglesias, sin embargo, en algunas de ellas, gracias a características como la libre interpretación de los textos bíblicos y la autonomía de las iglesias locales, ha sido posible que se lleven a cabo algunas ordenaciones pastorales en mujeres, debido a vacíos en las normatividades, a la creación de nuevos procesos o a la separación de sectores de las mismas iglesias. De acuerdo a las trayectorias estudiadas, aunque en su mayoría se trató de procesos atípicos de ordenación, a diferencia de la Iglesia católica, cuya posibilidad es inexistente desde la propia institución, en las Iglesias cristianas históricas es una posibilidad tangible.

De esta manera, se realizaron 11 entrevistas a profundidad a mujeres de denominación bautista, católica, luterana, metodista, presbiteriana y una autodenominada ecuménica, así como a cinco varones que pertenecen a estas mismas denominaciones, bautista, católica, luterana, metodista y presbiteriana. Estas últimas se llevaron a cabo con el fin de encontrar elementos comunes y específicos dentro de los procesos de ordenación, así como información relevante en la comprensión de las estructuras eclesiales de cada denominación.

El número de entrevistas realizadas obedece a que en México no existen muchas mujeres ordenadas como ministras de culto. Incluso dentro de los registros oficiales de asociaciones religiosas, o aquellos dedicados a la estadística de los grupos religiosos, no existen datos acerca de las mujeres ordenadas. En la mayoría de los casos, las entrevistadas son de las pocas mujeres ordenadas en México que pertenecen a sus respectivas denominaciones, si no es que las únicas de su denominación; en el caso de las católicas, aunque 
existen numerosas congregaciones de religiosas consagradas, la selección de las participantes obedeció a que se trata de mujeres que desempeñan cargos o trabajos sobresalientes y provistos de mayor autonomía dentro de sus propias congregaciones y que han realizado votos perpetuos de obediencia, castidad y pobreza.

En resumen, la elección de todas estas pastoras y religiosas respondió al hecho de que tienen una participación destacada dentro de sus iglesias y congregaciones, es decir, una práctica religiosa diferente a la que tradicionalmente tienen las mujeres (labores de servicio, cuidado y evangelización) dentro de las Iglesias cristianas y una sobresaliente y activa participación en el ámbito pastoral y religioso, académico y/o político social, reconocido mediante procesos institucionales, o bien, reconocido dentro del campo religioso y político social tanto por sus congregaciones como por sus pares.

Así, el objetivo de la presente investigación es conocer la experiencia y la vivencia religiosa respecto al papel de las mujeres dentro de las iglesias de credo cristiano en cuanto a las posiciones ocupadas, las labores desempeñadas y las relaciones de poder en que están inmersas, elementos que, sin distingo de institución religiosa, corresponden a una situación de desigualdad compartida por todas las entrevistadas. (Veáse en la página siguiente: Tabla 1. Participantes de acuerdo a la denominación religiosa y Tabla 2. Datos de la muestra al momento de las entrevistas). 
Tabla 1. Participantes de acuerdo a la denominación religiosa

\begin{tabular}{|c|c|c|c|c|}
\hline Credo & Grupo & Denominación & Congregación & Posición \\
\hline Cristiano & Católico & Católica & $\begin{array}{c}\text { Filipenses } \\
\text { Scalabrinianas } \\
\text { Santa Teresa del Niño } \\
\text { Jesús }\end{array}$ & Religiosas consagradas \\
\hline Cristiano & $\begin{array}{l}\text { Iglesias } \\
\text { históricas }\end{array}$ & $\begin{array}{c}\text { Bautista } \\
\text { Luterana } \\
\text { Presbiteriana } \\
\text { Metodista } \\
\text { **Ecuménica }\end{array}$ & & Pastoras ordenadas \\
\hline
\end{tabular}

**No es parte de las Iglesias históricas, sin embargo, es una pastora de origen metodista que se autodenomina ecuménica y cuya ceremonia de ordenación se realizó con la participación de pastoras/os de diferentes denominacioneS.

Tabla 2. Datos de la muestra al momento de las entrevistas

\begin{tabular}{|c|c|c|c|c|}
\hline Denominación & Edad & Estado civil & Hijas e hijos & Escolaridad \\
\hline Bautista & 58 & Casada & 2 & Licenciatura* y Maestría* \\
\hline Católica & 43 & Soltera & - & Licenciatura en sociología \\
\hline Católica & 46 & Soltera & - & $\begin{array}{l}\text { Licenciatura en comercio } \\
\text { Internacional }\end{array}$ \\
\hline Católica & 45 & Soltera & - & $\begin{array}{c}\text { Licenciatura, Maestría y } \\
\text { Doctorado }\end{array}$ \\
\hline Luterana & 46 & Casada & 2 & $\begin{array}{l}\text { Licenciatura en historia y } \\
\text { Licenciatura y Maestría* }\end{array}$ \\
\hline Luterana & 33 & Separada & - & Danza/Licenciatura* \\
\hline Metodista & 39 & Soltera & - & $\begin{array}{c}\text { Licenciatura en contabilidad } \\
\text { y Licenciatura* }\end{array}$ \\
\hline Presbiteriana & 43 & Casada & 2 & $\begin{array}{c}\text { Licenciatura en } \\
\text { administración, Licenciatura } \\
\text { y Maestría }\end{array}$ \\
\hline Presbiteriana & 50 & Divorciada & 2 & Licenciatura* \\
\hline Presbiteriana & 52 & Soltera & - & Licenciatura* \\
\hline Ecuménica & 56 & Divorciada & 2 & $\begin{array}{c}\text { Licenciatura en periodismo y } \\
\text { Licenciatura* }\end{array}$ \\
\hline
\end{tabular}

* Corresponden a estudios dentro del ámbito religioso, ya sea dentro de los seminarios de cada iglesia o en instituciones de formación religiosa a nivel de posgrado sobre teología, Biblia, divinidades, teología sistemática, etcétera. 
IGUALDAD, JUSTICIA Y TRABAJO

La igualdad para las mujeres dentro del campo religioso es un tema que puede ser tratado a diferentes niveles. La ordenación de las mujeres y su trabajo religioso es sólo una pequeña parte de lo mucho que se requiere abordar sobre su ejercicio y práctica religiosa en un campo que tiende a sustentar mediante su estructura institucional la desigualdad de las mujeres desde argumentos de Tradición o de fe. Los aspectos que se requieren considerar para hablar de la igualdad de derechos para las mujeres en este campo no sólo se limitan a la ordenación, sino también a una amplia gama de derechos, como son los derechos sexuales y reproductivos, los derechos laborales, los derechos a la formación académica, los derechos a la participación y representación y a la no violencia, por sólo mencionar algunos de los derechos humanos que deberían estar presentes de manera clara en la vida cotidiana de las mujeres dentro de las congregaciones e instituciones religiosas.

La construcción de la igualdad en este campo puede ser visibilizada a través de la participación de las mujeres mediante el trabajo que cada una de ellas realiza y la experiencia que tienen al respecto. El trabajo pastoral y religioso es el catalejo que hace posible ver de cerca, aquello que se muestra lejos y encubierto, aquello que bajo argumentos emanados de la supuesta existencia de una divinidad que se erige masculina y patriarcal, sirven de justificación para la desigualdad, asignando a la vocación y al servicio valores diferente de acuerdo al género de las y los agentes que participan dentro del campo. Las condiciones en que estas mujeres realizan su trabajo pastoral y religioso en nuestro país, permite evidenciar las diferencias producidas por el género y el entramado de las relaciones de poder en que están inmersas.

Para Nancy Fraser, la desigualdad de género está enraizada en una estructura económica que no brinda recursos suficientes y necesarios para las mujeres, en una diferenciación de estatus y en su falta de representación política. A través de su modelo tridimensional, la autora plantea cómo la redistribución, el reconocimiento y la representación son dimensiones desde las cuales se puede mirar la justicia social. El trabajo es uno de los medios con los que mujeres y hombres pueden participar de una vida social, política y económica, que además permite el intercambio de bienes como producto del mismo, lo que lo convierte en un recurso fundamental.

La igualdad es un principio fundante de la ciudadanía, es la posibilidad, al menos establecida de manera formal, de ejercer derechos y responsabilidades como sujetos políticos. Sin embargo, cuando miramos más allá de una igualdad establecida formalmente encontramos que existe una "contradicción entre los principios universalistas y la imposibilidad de realizarlos" (Lamas, 2001: 13) y que se trata de una igualdad formal que hace referencia a las mismas oportunidades, no a una igualdad identitaria, como menciona Marcela Lagarde, sino axiológica, es decir, la que se basa en igual valía para las personas (Lagarde, 1996: 207). Por otro lado, Elizabeth Jelin menciona que es necesario tener cuidado, dado que cuando la igualdad está sustentada en un derecho universal basado en la naturaleza, podemos correr el riesgo de "una formalización excesiva de los derechos, 
aislándolos de las estructuras sociales en que existen y cobran sentido" (Jelin, s/f).

La igualdad, entonces, no es un concepto dado, ahistórico, estático o lineal; por el contrario, la construcción de la igualdad debe mirarse como un proceso que debe ser analizado y construido no sólo desde su contexto estructural y/o institucional y legal, sino también desde una perspectiva cultural, diversa y subjetiva de sus actores.

$\mathrm{Al}$ interior de estas instituciones religiosas, las mujeres conforman y participan de las estructuras llevando a cabo funciones que mayoritariamente son una extensión de las labores realizadas en el espacio doméstico y relacionadas con su condición, situación y posición de género (Lagarde, 1996), como la enseñanza a niños y niñas, el cuidado de enfermos y labores de asistencia en general, por mencionar sólo algunas. Sin embargo, el acceso a puestos jerárquicos y reconocidos institucionalmente, como son los ministerios ordenados, no es para ellas una posibilidad real, y aun cuando escalan y logran ejercerlos, no lo hacen en condiciones de igualdad en cuanto a los procedimientos para ser reconocidas y legitimadas: las condiciones laborales en las que los desempeñan no se caracterizan por horarios de trabajo y remuneraciones justas, y siempre están sujetas a la doble evaluación de su trabajo y a la constante negociación con las jerarquías eclesiales, por mencionar algunas limitaciones.

Yo recuerdo un caso de una chica, por ahí de los años 79 u 80, una mujer que la dejaron hacer todo en el seminario metodista, la dejaron hacer todos los estudios, terminó su tesis, se tituló con honores, a la hora de asignarle una iglesia le dijeron: no, es que aquí no hay pastoras. Como 20 años después, tuvieron una Obispa (Pastora Ecuménica, 56 años).
La desigualdad, por lo tanto, no sólo puede ser entendida como la negación de una igualdad manifestada en las diferencias sociales, económicas y políticas, sino también como "asimetría que en las diferentes sociedades se manifiesta en marginación, subordinación y participación inequitativa ante los derechos de ciudadanía" (Vélez, 2006: 379). Para Lagarde, la desigualdad es una forma de discriminación que se basa en la creencia de que algunos seres humanos o grupos tienen un valor inferior a otros, y por lo tanto la justicia, como principio ético, es lo que sustenta la reivindicación de la igualdad como un principio normativo y como un derecho (1996: 207).

Desde la perspectiva tridimensional de Nancy Fraser (2008), la justicia requiere de la redistribución, el reconocimiento y la representación como reivindicaciones económicas, culturales y políticas. Lo que se traduce en una distribución más justa de recursos, la aceptación de la diferencia sin que ésta sea sinónimo de desigualdad de estatus y la inclusión dentro de la vida social y política desde la propia identidad sustentada en la idea de una igualdad de derechos, así como de la aplicación de un marco institucional que los respalde.

Bajo este supuesto acerca de la importancia de los recursos, el reconocimiento y la representación como un principio que posibilita la igualdad, podemos voltear la mirada hacia la manera en cómo las mujeres dentro de los ámbitos religiosos cristianos son reconocidas, legitimadas y remuneradas en aquellos aspectos relacionados con el trabajo pastoral o ministerial religioso que realizan, sobre todo cuando desempeñan funciones 
dentro de espacios que tradicionalmente no han sido ocupados por ellas y que incluso llegan a tener cierta categoría jerárquica dentro de sus iglesias, a diferencia de su participación como congregantes o encargadas de la formación de niños y niñas o de las funciones asistencialistas en las que ellas siempre son la mayoría.

Se utiliza el término trabajo, y no servicio o labor, para hacer la distinción de que se trata de una actividad que está determinada por un tiempo, con funciones establecidas y que origina un producto de dicha actividad de tipo social y/o económico que permite algún intercambio de bienes. Asimismo, se entiende como trabajo pastoral o ministerial religioso el acompañamiento a los integrantes de sus congregaciones o a grupos específicos como parte de las funciones que conciernen a su ministerio religioso, en el que desempeñan funciones de preparación y celebración de cultos y liturgias, apoyo, contención y acompañamiento emocional, visitas, búsqueda y facilitación de recursos para otros, educación, formación y evangelización, administración y cuidado de los recursos de la iglesia. En algunos casos, también comprende el trabajo específicamente políticosocial a través del vínculo con otras instituciones.

El reconocimiento, la legitimidad y la remuneración hacia su trabajo, así como las condiciones en que lo llevan a cabo, ponen de manifiesto la disparidad existente, tanto con los varones que ocupan los mismos puestos, como entre las mujeres de acuerdo a la denominación a la que pertenecen y su relación con la clase, la formación académica y su estatus.
Cada Estado tiene presbiterios que pertenecen a la Asamblea General. Y los presbíteros te decían: sí te pagamos, pero vete a la escuela bíblica; entonces, las mujeres, es mi experiencia y la de una de mis compañeras en el seminario, recurríamos a otras formas [para solventar los gastos]. Por ejemplo: yo hice el aseo de mi maestra de psicología del seminario por tres años para pagar mi licenciatura en teología. Trapeaba, mientras mis compañeros en el presbiterio, tenían la beca completa para libros, para colegiaturas, etcétera, por ser varones (Pastora Presbiteriana, 50 años).

Por otro lado, también las nuevas formas en que las mujeres realizan su ministerio religioso nos muestra un cambio en los significados de su actividad en relación con su posición de género. La manera en cómo participan desde otros lugares dentro de este campo abre incluso la posibilidad de reelaborar nociones sobre participación y derechos y descolocarse de la idea de que este trabajo, en su caso, es sólo una vocación traducida en amor y cuidado al servicio de las y de los otros, para convertirse en una participación más activa en la toma de decisiones, en liderazgos más horizontales o en el autorreconocimiento de la importancia del trabajo desempeñado. Lo anterior, contrario a la idea tradicional de vocación religiosa que en muchas ocasiones es esencializada y naturalizada a través de la exaltación de imágenes religiosas femeninas que muestran una incondicionalidad y disponibilidad permanente.

Es el caso de las mujeres entrevistadas dentro de las Iglesias bautista, luterana, metodista y presbiteriana que han conseguido cierto reconocimiento y legitimación a través del ejercicio de un ministerio ordenado, así como de religiosas 
consagradas pertenecientes a la Iglesia católica cuyo ministerio es ejercido a través del activismo o la inserción en campos laborales que tradicionalmente no estaban destinados para ellas (trabajo académico y político-social).

Para mí, el ser pastora es estar en espacio de diálogo, de constante diálogo, de constante provocación... Yo creo que para hablar nuevamente de inclusión hay que eliminar las barreras y ya... Para mí, el ser pastora no tiene otro significado más que eso, la participación en la congregación.

Abrir brecha para hacer nueva práctica eclesial, para hacer una nueva labor pastoral, el vernos como y entendernos como pastoras y pastores no como ejemplos de vida, sino como personas comunes y corrientes con todas sus debilidades, con sus problemáticas, con las problemáticas que tiene cualquier persona y que en base a ellas también podemos trabajar como pastores, que eso no nos impide ser pastores, que las fragilidades no nos impide trabajar; al contrario, poner esas heridas al servicio de los demás, de alguna forma y dejarnos también pastorear por nuestra congregación (Pastora Luterana, 33 años).

A partir de sus experiencias, se abordan a continuación algunos aspectos sobre el trabajo pastoral o ministerial religioso y las condiciones en que éste es llevado a cabo desde la propuesta teórica del modelo tridimensional de Nancy Fraser.

\section{Reconocimiento, representación y redistribución}

En cuanto al reconocimiento, es decir, al valor que se otorga a una condición o situación identitaria y que tiene una relación directa con la manera en la que el individuo se concibe dentro de un entorno social y político (Fraser, 2006), el trabajo pastoral y eclesial como elemento unido fuertemente a su identidad religiosa que realizan las pastoras de estas Iglesias y las religiosas consagradas en México, es un trabajo gravemente invisibilizado. Esto se debe en parte porque se encuentra dentro de un ámbito de la vida que se considera privado e incluso místico, en el que no es posible acceder sin ocasionar tensiones, dada la relación simbiótica divinidad-iglesia-jerarquía, pues al cuestionar a una sobre las condiciones de las mujeres, se cuestiona al mismo tiempo a las dos restantes, y con ello los argumentos que desde la Tradición se dan para justificar la no inclusión de las mujeres en los puestos de poder y toma de decisiones dentro del campo religioso.

[...] Porque debido a la separación entre espacios bien definidos mediante el dualismo entre la casa familiar y el ámbito público (hogar-calle), las mujeres siempre han estado como "de visita" en el templo, espacio privilegiado de los hombres porque su "zona de poder" es la casa, el hogar, es decir, la domesticidad y la invisibilidad, y el de los hombres, la calle, el poder público y visible. El lugar de ellas seguiría siendo "el atrio del templo", las afueras, para estar a la vista y bajo la supervisión de sus señores.

[...] Y eso es lo que se sigue practicando en nuestro medio eclesial: un liderazgo, en este caso, sancionado por la ordenación, basado en la dominación y la falsa idea de superioridad. Nada más inaplicable en el caso del servicio cristiano, si se recuerda una vez el modelo de Jesús, quien se abajó a sí mismo para servir únicamente. Por ello, lo que hoy se requiere son verdaderos cambios estructurales en la Iglesia que tengan que ver con la despatriarcalización de todos los ministerios, y no solamente administrativos ni burocráticos. De otra manera, el dilema consistirá, ahora, de aceptarse la ordenación femenina, en cómo ser una ministra en un mundo patriarcal (CervantesOrtiz, 2013).

También porque en el ser-para-los-otros de las mujeres (Basaglia, 1983, citada en Lagarde, 1990) el cuidado y la participación no son consideradas una labor sino un deber o una esencia. Y por último, porque aun cuando asumiendo posiciones diferentes, de mayor jerarquía institucional o moral y en algunos casos un liderazgo identificado, 
el trabajo realizado no se pondera con igual valía y muestra de ello es la constante sobreevaluación y escrutinio al que es sometido su ejercicio ministerial.

$\mathrm{Al}$ ingresar al seminario, estaba el currículo de clases. Tomabas las mismas clases: hebreo, griego, exégesis bíblica, hermenéutica, etcétera. Todo, todo, todo era igual para mujeres y hombres. Nosotras fuimos de las primeras en estudiar en los seminarios [...] en cuanto a conocimiento, teníamos el mismo conocimiento. Salían quince o veinte varones y una mujer... y aún sigue ese proceso.

Nosotras, al ingresar al seminario, sabíamos ciertamente que al salir del seminario, cuál iba a ser nuestra perspectiva de trabajo, estaba de la jodida, porque no nos iban a dar chamba, en ninguna área pastoral (Pastora Presbiteriana, 50 años).

La mayoría de las pastoras entrevistadas fueron ordenadas en situaciones diferentes a los procedimientos normalmenteseguidosporsuspares varones: fueron cuestionadas en su capacidad para desempeñar la labor pastoral y debieron cumplir con requisitos que otros pastores no tuvieron que cumplir. Asimismo, una vez ordenadas, su trabajo es el elemento constantemente evaluado y comparado. El reconocimiento de su trabajo proviene, con mayor frecuencia, de los sectores seculares, de sus congregaciones con las cuales han hecho una labor de convencimiento y formación, y de sus relaciones interpersonales más cercanas, no así de las instancias eclesiales institucionales. Cabe decir que la Iglesia Metodista de México y la Iglesia luterana son las únicas que cuenta con una normatividad que, de manera general, contempla procedimientos menos discrecionales para mujeres y hombres en cuanto al proceso que las personas que quieren llegar al pastorado deben seguir.
En las siguientes narraciones se muestra cómo en ocasiones la labor para llegar a la ordenación fue de tipo congregacional, como en el caso de la Pastora Bautista, mientras que en otras, como en el caso de la Pastora Metodista, fue una posibilidad ofrecida desde la misma institución.

Desde que empezamos la congregación, empezamos a platicar de una equidad e igualdad entre el pastorado de mi esposo y el mío [...] En mi comunidad local hubo una preparación, porque un año estuvimos trabajando desde los niños para que comprendiéramos qué es lo que iba a pasar en un año, íbamos a ser una Iglesia bautista que iba a tener una mujer, que iba a ser pastora, que en otras iglesias no había mujeres reconocidas como pastoras; que el pastor y yo podíamos hacer lo mismo, dar la palabra o la comunión, la Eucaristía o Santa Cena como le decimos nosotros, bautizar... digo, elementos sagrados que históricamente solamente [hacían] los hombres. Entonces los niños toman una nueva pedagogía, es decir, vamos a ser un grupo nuevo y diferente porque creemos que es lo que Dios nos está llamando a hacer (Pastora Bautista, 58 años).

Nos consideran a todos los que salimos, independientemente del género, nos consideran como "probandos", toda aquella persona que entra al seminario tiene que..., mientras siga estudiando el seminario, tener cuatro años de prueba y así nos llaman; entonces "pastores a prueba" y durante esos cuatro años pues debe uno de ser constante y probar que realmente tenemos "el llamado" del pastorado y que sabemos ejercerlo (Pastora Metodista, 39 años).

En cuanto a las religiosas consagradas cuya ordenación no es posible dentro de la Iglesia católica como ministras de culto, el reconocimiento hacia el ejercicio de su ministerio se encuentra sobre todo en el ámbito académico o en el trabajo político social que realizan dentro de las asociaciones civiles y ciudadanas. Se trata de un reconocimiento velado, enmascarado dentro de discursos de austeridad que contrastan con una participación activa e incluso 
directiva dentro de sus ámbitos de trabajo, donde se pueden negociar o tomar algunas decisiones siempre y cuando no nombren de manera explícita su participación como atributo de reconocimiento personal (el trabajo que realizan y el reconocimiento otorgado al mismo se expresa como colectivo y se valida cuando se trata de acciones dirigidas a terceros), pues de lo contrario podrían ser cuestionados valores altamente estimados en este ámbito como la obediencia o la humildad.

[...] estuve como secretaria ejecutiva a nivel nacional de la Conferencia Episcopal Mexicana, en el tema de movilidad humana; y bueno, haber hecho esta experiencia y todo, nos tocó prácticamente ver este salto cualitativo y agresivo que sufrió la migración en todos los sentidos.

Desde la Conferencia Episcopal Mexicana, yo tengo muy claro y tenía muy claro en aquel momento, entre más manos nos ayuden como congregación, súper bien, pero no es mi trabajo, no es mi legitimación, es que necesito que todas estas manos y todas estas personas sean reconocidas como el grupo humanitario que acompaña a las personas. Yo creo que avanzamos mucho en ese aspecto, hay figuras muy públicas y hay figuras que nacen de este trabajo operativo donde la presencia, no sólo de mi persona, sino de la oficina, que en su momento éramos la base, la base que sostenía toda esta red de trabajo estábamos siempre detrás pero siempre detrás y adelante, no visibles, sino adelante en el sentido de decir ¡ah! es que esto va para acá, nosotros tenemos que ir por aquí; no decidíamos porque hemos hecho siempre un trabajo muy consensado, cuando es posible consensarse y vamos siempre como al frente ¿no? Creo que eso ha sido la legitimización de, vuelvo a insistir, no es sólo de mi persona, del equipo con el que continúo trabajando (Religiosa Consagrada, 46 años).

El trabajo pastoral es un trabajo que determina el tiempo, el espacio y las relaciones interpersonales de estas mujeres. Es un trabajo que se encuentra cruzado por la desigualdad debido al género, por las relaciones de poder y estructuras jerárquicas androcéntricas en las que están inmersas y las cuales no consideran su trabajo en la misma posición que la de sus pares varones y la diferencia, entonces, es sinónimo de su desigualdad de estatus.

La segunda dimensión que Nancy Fraser hace respecto a la justicia es la representación. Se trata de una noción de inclusión dentro de la vida social y política desde la propia identidad basada en el supuesto de estar representada legal y políticamente dentro de la comunidad y sociedad a la que se pertenece (Fraser, 2008: 42). Este aspecto es más complicado de revisar dado que podría pensarse que la representación sólo es posible desde su inserción en la vida secular como ciudadanas, dado que aunque participan en la vida política y social desde su identidad religiosa, este no se considera un espacio en el que abiertamente deban estar representadas como mujeres de Iglesia. Ahora bien, si consideramos a las Iglesias como instituciones y limitamos la representación al contexto religioso, podemos encontrar que las mujeres no están representadas (cuando lo están) en condiciones de igualdad. No obstante, en la mayoría de los casos no existe ninguna representación femenina dentro de las estructuras eclesiales en puestos relacionados con la toma de decisiones y el ejercicio del poder.

Cuando ya son pastores, para todos son pastores y son los máximos, son los mejores, el obrero está acá y aparte las misioneras hasta por acá [señala grados en descenso a través de movimientos de manos] [...] entonces entré como obrero, entonces estaban así los pastores y así nosotros, pero las misioneras están todavía más abajo.

Sólo los que entrecomillas saben más, son los que están en mejor puesto, tienen mejor sueldo, tienen el lugar en la ciudad y nosotras vámonos al campo (Pastora Presbiteriana, 52 años). 
Como se mencionó anteriormente, en México, Iglesias como la metodista o luterana, consideran la ordenación de las mujeres desde hace algunos años; en otras como la Iglesia bautista o presbiteriana, la ordenación de mujeres implicó rupturas o separaciones y la conformación de nuevas congregaciones. De cualquier manera, el que algunas mujeres dentro de estas Iglesias hayan sido ordenadas no significa que el número de ordenaciones de mujeres se equipare al número de ministros varones ordenados, o que los procesos para llegar a la ordenación se hayan dado dentro de una igualdad tanto de normatividades como de circunstancias, ni que las condiciones en que realizan su trabajo sean justas.

Se propuso y exigió que se hiciera la votación, porque era el momento de la inclusión de la mujer en la Iglesia presbiterana, y se hizo a nivel nacional. Hubo una votación del $90 \%$ de pastores en contra de la ordenación de la mujer, mínimo de 3\% a favor, así se hizo el veredicto del "no" a la ordenación. Entonces, los siete pastores que hicieron manifestaciones públicas en los presbiterios, para decir que se verificara el texto bíblico, que se analizara, fueron excomulgados (Pastora Presbiteriana, 50 años).

La siguiente narración nos permite identificar dentro de una Iglesia como la metodista que cuenta con mayores espacios de representación para las mujeres incluyendo la ordenación, las diferencias al interior de las labores que éstas realizan y la jerarquización existente entre los mismos puestos que ellas ocupan. Es importante mencionar que se trata de una excepción, pues en el resto de las Iglesias la distribución de puestos no es tan clara dentro de la estructura institucional eclesial, ni cuenta con tantos puestos ocupados por mujeres.
La Iglesia Metodista de México es, incluso, la única que cuenta con una Obispa.

La estructura de esta Iglesia da un ejemplo de cómo la organización institucional de las Iglesias es un factor clave en la construcción de la igualdad para las mujeres, porque cuando desde la propia institución es posible acceder a otros puestos se abre una posibilidad de transformación de la praxis religiosa y de cambio en la estructura eclesial.

En la Sociedad Misionera Femenil, trabajaban anteriormente, por decir, antes de los noventas, trabajaban los talentos: coser, tejer, hacer manualidades y después venderlas. Regularmente las ventas con los talentos junto con las ofrendas permite tener una serie de participaciones en niveles más altos o en su comunidad para apoyar trabajo de pastoras, antes eran diaconisas. Entonces, el trabajo de una pastora, el desarrollo de una congregación, tienen lo que llamamos cajitas de bendición, que son también ofrendas que van guardando de lo que va quedando de su gasto y lo ocupan o lo llevan a la administración de su organización, a nivel conferencial, para desarrollo de proyectos.

Desde que llegué a la Iglesia ha sido grato saber que hay pastoras; tenemos pastoras, superintendentes, aquí tenemos una en la Conferencia de Baja California, en Sonora hay dos, en la de Puebla ha habido superintendentes mujeres también, en la zona centro del país de Jalisco y al norte de Veracruz también hay varias mujeres, creo que son dos o tres mujeres las que están. Primero había diaconisas, las cuales, por norma, venían y estudiaban en la escuela para diaconisas, tres años, después tenían que trabajar en la iglesia, mínimo dos años antes de casarse. Cuando la diaconisa se casaba, dejaba el trabajo del ministerio. Vienen los tiempos y se logra la orden, el cambio del concepto, ya no son diaconisas: pueden ser presbíteras, primordialmente presbíteras locales que son para una región pequeña y después también viene la oportunidad de que sean presbíteras itinerantes. De hecho, nuestra Obispa, que fue Obispa, y otra pastora en sureste que terminó sus funciones como superintendente (Obispo Metodista).

En el resto de las Iglesias la representación de las mujeres se encuentra de manera mayoritaria 
sólo a través de las ligas y grupos femeniles, o bien en algunas reuniones donde se presentan informes respecto a labores de formación y evangelización en grupos de infantes y jóvenes o la rendición de cuentas respecto a la dirección de algunas escuelas.

En otras Iglesias como la luterana se realizan reuniones anuales de mujeres en las que también son mujeres las que llevan la coordinación y agenda de las reuniones. Sin embargo, en la mayoría de los casos, el tipo de representación al interior del campo religioso se da en medio de una separación genérica que impide la participación de las mujeres en la toma de decisiones sobre las agendas generales de las instituciones eclesiales y que las limita a una injerencia nuevamente relacionada con los grupos de los que siempre se han encargado (infantes y mujeres). Estas reuniones presentan informes y resultados que son integrados a los trabajos de la Iglesia, pero generalmente se dan en una sola dirección, pues ellas no están representadas ni en cantidad, ni en posiciones de poder que les permitan participar y decidir sobre los acontecimientos más importantes en el rumbo de las Iglesias. Se trata de una representación desde la periferia en la que sobre ellas y su participación hay normatividades $\mathrm{y}$ procedimientos que establecen el tipo de contacto, responsabilidad y papel que juegan dentro de la estructura.

Su trabajo, por lo tanto, queda representado con una valía disminuida que se diluye en la falsa idea de que no necesitan este tipo de representación y poder dentro de la institución porque su labor es importante y no busca ser destacada pues se trata de actos desinteresados.
Para las religiosas católicas la representatividad desde esta perspectiva no es una posibilidad dentro de la institución. La responsabilidad para cubrir otros cargos puede darse cuando se trate de una petición institucional directa, relacionada con las capacidades personales e inocultables de la religiosa que requieren que ella sea la encargada de alguna labor determinada. Sin embargo, el reconocimiento de esta labor no tiene impacto en la representatividad de las mujeres dentro de la institución. Las mujeres no están representadas en la estructura eclesial desde ningún puesto de poder y tal vez es en la Iglesia católica donde esto se hace más evidente.

Por último, hablaré de la redistribución, es decir, la distribución justa de los recursos. Con ello, abordaré más ampliamente los resultados de investigación relacionados con el trabajo pastoral o ministerial religioso y su relación con el nivel educativo de estas mujeres, puesto que éste es, tal vez, uno de los principales recursos para la obtención de capital que puede ser parte del intercambio de bienes al que Pierre Bourdieu hace referencia en su texto Estructura y génesis del campo religioso (Bourdieu, 2006).

El trabajo de estas mujeres, tanto de las que han sido ordenadas por sus Iglesias como de las consagradas dentro de sus comunidades religiosas o aquellas que están en el proceso de ordenación, no se realiza en un horario establecido formalmente o con una remuneración basada en el número de horas trabajadas o en las funciones desempeñadas. La mayoría de ellas, además de las horas dedicadas al trabajo pastoral, desempeña otros trabajos también relacionados con la formación religiosa o la participación social y política como una forma de 
completar el ingreso y, en la mayoría de los casos, también una forma de cumplir con el carisma de sus congregaciones o con los propósitos y acciones que consideran dan sentido a su llamado religioso.

Pues es como todo el año, las 24 horas. Fíjate, antes había un concepto de pastor de medio tiempo y pastor de tiempo completo, o sea yo creo que soy pastora de tiempo completo, aunque tenga otras actividades... Porque tienes tu carga, tu pensamiento también siempre, o sea, todos los días, nunca falta todos los días alguien que tengas que visitar, a veces alguien que te escribe un correo o te hable por teléfono, entonces yo creo que el trabajo pastoral es algo de tiempo completo porque es parte de tu vida (Pastora Metodista, 39 años).

Para Laís Abramo (2004) en el mantenimiento y la reproducción de las desigualdades existentes entre hombres y mujeres en el mundo del trabajo empresarial, inciden varios factores derivados de una división sexual del trabajo basada en un orden de género que asigna a la mujer la función del cuidado del mundo "privado" y de la esfera doméstica, y le atribuye a ésta un valor social inferior al mundo "público", y en el que se desconoce también su valor económico.

Para ella el imaginario social construido a partir de la dicotomización del mundo en el que se atribuyen diferentes valores al trabajo de los varones como principales proveedores, en contraste con el trabajo de las mujeres como responsables del ámbito doméstico, es lo que reafirma la idea de las mujeres como una fuerza de trabajo secundaria o complementaria, entendiendo como imaginario social "el conjunto de visiones de sentido común, más o menos estructuradas y racionalizadas, que tienen los individuos en general, hombres y mujeres, sobre sus lugares, roles y funciones, en el trabajo, en la familia, en la sociedad, en la esfera pública y en la esfera privada" (Abramo, 2004: 227).

Para esta autora, la idea de fuerza de trabajo secundaria o complementaria dentro del campo empresarial parte del supuesto de que el trabajo es un ámbito secundario del proyecto de vida de las mujeres y que se percibe como eventual o inestable, motivo por el cual los ingresos generados por éste también son concebidos como secundarios.

Esto, como dice Abramo (2004), está relacionado con la organización de los roles de género y los marcos económicos que no incorporan a la actividad económica el trabajo no remunerado y que establecen limitaciones a las mujeres en el trabajo por una supuesta "naturaleza femenina" (maternidad, cuidados que impiden viajar o tener horarios nocturnos, etcétera) a la que los empresarios entrevistados en su estudio atribuyen la inestabilidad del trabajo de las mujeres y por lo que la consideran una fuerza de trabajo secundaria. Incluso en este imaginario empresarial, los entrevistados consideran que la razón por la que las mujeres están dispuestas a obtener menos ingresos que los hombres se debe a que no negocian sus remuneraciones, puesto que sus familias son la centralidad de sus proyectos de vida y que el trabajo no es una obligación.

Este argumento de corte esencialista encontrado en los empresarios entrevistados no es sólo parte de un imaginario empresarial; también lo es, como lo menciona esta autora, del imaginario social y, puede decirse, del imaginario religioso.

Dentro del campo religioso, el trabajo pastoral de las mujeres, ya sea desde los ministerios ordenados 
como en las religiosas consagradas, en la mayoría de los casos, no cuenta con la misma remuneración que el de sus pares varones ni es uniforme entre las mujeres de las denominaciones estudiadas. El trabajo religioso de las mujeres es también considerado secundario o complementario, pues el centro de sus vidas es la relación interpersonal con las y los otros desde el cuidado y el afecto; tiene un valor religioso inferior y se desconoce su valor económico, una muestra de ello es la desigualdad en los salarios y la dificultad de las entrevistadas para contabilizar el número de horas trabajadas y la remuneración que sería justa por el producto de su trabajo.

\section{Trabajo y formación académica}

Nuevamente cito a Abramo (2004) cuando considera que el incremento en la escolaridad de las mujeres ha sido un factor importante para mejorar las posibilidades y las condiciones de las mujeres en el trabajo, pues aumenta sus tasas de participación, sus ingresos promedio y sus posibilidades de acceder a un empleo formal (que amplia probabilidades de acceder a la protección social). Sin embargo, como ella misma menciona, los mayores niveles educativos no les garantizan a las mujeres más y mejores oportunidades de empleo en relación con los hombres. Ellas necesitan de certificados educativos significativamente superiores para acceder a las mismas oportunidades de empleo.

Los datos encontrados respecto a la formación académica de las entrevistadas resultan coincidentes con la información brindada con Abramo, sólo que además en el caso de las pastoras y religiosas, no sólo se requieren certificados educativos superiores sino una trayectoria religiosa desde estándares morales casi impecable que no deje duda de su capacidad para realizar el trabajo, sumado esto a la participación continua comprometida en sus congregaciones.

Como podemos observar en la Tabla 2, su alta formación académica, que abarca, además de los estudios religiosos de su especialización, otras disciplinas (periodismo, historia, comercio, contabilidad, sociología y danza) posiblemente sea uno de los factores que les ha permitido posicionarse en lugares diferentes dentro del ejercicio ministerial, así como construir nuevas formas de participación acercándose al lugar de los especialistas del saber religioso.

La formación académica superior ha sido un factor para poder acceder a un ministerio ordenado, o bien, para tener relativa autonomía en las decisiones y opciones de vida dentro de sus congregaciones religiosas, como es el caso de las religiosas consagradas que, a diferencia de otras integrantes de sus congregaciones, cuentan con cierta autonomía para negociar su lugar y sus condiciones de práctica religiosa.

Posiblemente, la formación en espacios seculares ha contribuido a modificar el estatus y, con ello, aspectos de la representatividad de las mujeres en el campo religioso. A través de su experiencia, podemos observar cómo su posición dentro de este campo, desde un lugar de autoridad o de autorreconocimiento de algunos derechos como el ejercicio ministerial/pastoral, o como mujeres con derechos en cualquier campo desde la vida secular, permite negociar algunos aspectos relacionados con la toma de decisiones y la autonomía, aunque esto no se vea reflejado en 
una revalorización, reconocimiento y una mejor remuneración de su trabajo.

\section{Trabajo, remuneración y prestaciones}

Los ingresos que las pastoras reciben como producto de su trabajo cuando han sido ordenadas por sus Iglesias mediante un proceso que contempla la pasantía o terminación de estudios teológicos, y que en su mayoría cuentan con una iglesia, es decir, que han sido signadas oficialmente a una iglesia como responsables y, por lo tanto, con una congregación, era de entre 2,500 y 12,000 pesos al mes al momento de la realización de las entrevistas. Como se puede ver, la diferencia entre el sueldo más bajo y el más alto es notoria. Regularmente, estos sueldos deben ser completados con otros trabajos o, en su caso, son un complemento de su remuneración laboral en otras instituciones. En los casos de las Iglesias luterana, presbiteriana y bautista, los ingresos son una contribución a la pastora determinada por la congregación, por el trabajo realizado. En otros casos, como en la Iglesia metodista, existe un tabulador de sueldos que considera el trabajo pastoral de fin de semana, de medio tiempo y de tiempo completo, así como el pago de servicios y la provisión de una casa pastoral.

Son autónomas las congregaciones, cada congregación le paga a su pastor. En la congregación percibo..., porque no es siempre lo mismo, pues un aproximado de 2,000 o 2,500 pesos.

En el seminario luterano también percibo un sueldo que tampoco es muy grande, pero bueno... En el seminario estoy en el área de los cursos en línea, soy administradora de las páginas, de crear cuentas, crear propaganda, ocuparme de cuestiones técnicas o cosas así. De ahí recibo 3,000 pesos (Pastora Luterana, 33 años).
En un país donde el salario mínimo equivale a 73 pesos diarios, 2,500 pesos mensuales no es un sueldo que vaya de acuerdo con el número de horas trabajadas, si consideramos que incluye la preparación de liturgias, acompañamiento de congregantes y funciones administrativas durante la semana y las horas dedicadas al culto el fin de semana.

En general, la remuneración que las pastoras reciben por su trabajo les es entregado directamente. Sin embargo, hay ocasiones en que el pago mensual puede ser entregado bajo el concepto de pareja pastoral o, incluso, sólo al esposo de la pastora, "dándose por entendido" que se trata del sueldo de ambos. Esto ejemplifica el difícil proceso de reconocimiento del trabajo de algunas de estas mujeres dentro de los ministerios, dado que muestra que, en ocasiones, su trabajo sólo es considerado como secundario o complementario, como una extensión del trabajo de los varones, que son quienes tienen el reconocimiento.

En el caso de los varones entrevistados dedicados al ministerio pastoral, el sueldo más bajo obtenido por este trabajo es de 10,000 pesos, y el más alto, de 12,000 , lo que nos permite ver el fuerte contraste.

En cuanto a la remuneración producto de actividades docentes, en caso de una religiosa y un sacerdote pertenecientes a la Iglesia católica, quienes poseen nivel de doctorado y que imparten clases en instituciones particulares, la diferencia entre sus sueldos equivale aproximadamente a 20,000 pesos.

¡Ush! Ésa es una buena pregunta, porque yo no tengo salario fijo, mira, depende de las académicas, yo diría que al menos así de general, al mes, son como 
$16,000[\ldots]$ doy la mitad a mi congregación (Religiosa Consagrada, 45 años).

Para las y los integrantes de esta Iglesia, un porcentaje de su remuneración mensual debe ser entregado a la congregación u orden a la que pertenecen; este porcentaje es de entre el 40 y $50 \%$ del total de sus ingresos; el resto es ocupado para gastos personales, en el caso de vivir dentro de la congregación, y para pago de renta, transporte, alimentación y gastos personales, en el caso de las religiosas que viven fuera de la casa congregacional.

No obstante, vivir fuera de la casa congregacional, si bien brinda cierta autonomía, también aumenta los gastos económicos mensuales, lo que probablemente requiere de mayores negociaciones sobre el ingreso en cuanto a la distribución de recursos con quien está a cargo de la administración de estos, como lo son las "ecónomas", ${ }^{4}$ con lo que también se adquieren ciertos compromisos y sujeciones de otro orden además del económico.

Del sueldo oficial que gano, todo tiene que entrar, pero hay otros apoyos que salieron en el camino, como yo no tengo que entregar cuenta de eso, yo me quedo con eso y a veces me piden cuentas y a veces no, entonces son, por ejemplo: para uso de pasajes, de teléfono, de copias. Hay ocasiones en que no se gasta todo y, por ejemplo: si es para teléfono, es mi teléfono entonces yo siempre tengo ese servicio y de eso ya no tengo que dar cuentas, porque, es como si hubiera más conciencia con las hermanas.

En la distribución de los bienes no tendrías que estarles pidiendo, se haría un balance: necesitas tanto... y si ocupas más, pues nos avisas...

Y no, o sea, yo cada... me dan 200 para pasajes y cada que se me acaba les tengo que estar pidiendo... y así, y así y... jay porque te lo acabaste tan pronto!

4 Mujeres encargadas de la administración y distribución de recursos al interior de cada congregación religiosa femenina.
Bueno, antes me causaba mucho problema, ¡ay, pero que ya no quieren dar!... pero no sé qué. Ahora digo: no, pues se acabó, se acabó y me lo tiene que dar porque es un dinero de todas, no es su dinero (Religiosa Consagrada, 43 años).

Para quienes pertenecen a otras congregaciones y realizan trabajo político-social, el sueldo que reciben por sus actividades dentro de asociaciones de la sociedad civil oscila entre los 6,000 y 11,000 pesos. En el caso de estas religiosas católicas, el total de sus ingresos es entregado a la congregación y les es devuelto en forma de una mensualidad ya determinada con base en un presupuesto sobre transporte, alimentación, renta y gastos personales, mismo que en algunos casos debe incluso ser comprobada o renegociada.

Entonces eso que ganamos, no lo gastamos personalmente, sino que entra a un fondo común donde sale tu ropa, medicina, alimento; si se puede, se hace un ahorro y también de allí después surge una cuenta, cada casa da cuentas a nivel nacional e internacional. Si por alguna razón, alguna casa tiene necesidad económica se le ayuda, se la da por decir un sueldo de la casa general, y si vienen imprevistos, como cuestiones de salud, operaciones o un proceso largo de atender alguna enfermedad, también hay una solidaridad nacional y una solidaridad internacional. Pues hay quien decide qué actividades, qué recursos, cada cuánto, por ejemplo, tienes un trabajo de diario, se hace un balance de pasajes, de acuerdo al lugar (Religiosa Consagrada, 43 años).

Las prestaciones recibidas por este trabajo son mínimas. Sólo dos pastoras cuentan con servicio médico, una de ellas con un seguro médico que es otorgado por la congregación, y otra por parte de la iglesia a la que pertenece y que contempla para las pastoras de medio tiempo seguro social. El resto no cuenta con servicio médico por el trabajo 
pastoral; las Iglesias no consideran estos aspectos (excepto la Iglesia metodista). Algunas de ellas tienen seguridad social debido a otros trabajos que realizan o, en algunos casos, como el de la Iglesia católica, cuando enferman, la congregación paga los gastos médicos. Otras recurren a tratamientos alternativos, o como dijo una de ellas durante la entrevista: "casi no me enfermo".

Yo tengo seguridad social... en la conferencia de religiosas y también por parte de mi congregación. Normalmente sí [es] para todas las que trabajamos, las que estamos en activo. Las que se han retirado, ya están jubiladas o que han trabajado como académicas también en otros espacios, están jubiladas y tienen la seguridad social. Hay hermanas que no tienen seguridad social porque no han ejercido un trabajo académico y compran un seguro para ellas (Religiosa Consagrada, 45 años).

Esta situación, al igual que la posibilidad de una jubilación con derecho a una pensión, o la posibilidad de adquirir una vivienda, es algo que queda fuera del entorno laboral de estas mujeres, pues su trabajo adquiere el carácter no sólo de invisibilidad, sino también de informalidad, lo que las deja sólo limitadas en el ejercicio de sus derechos ciudadanos en relación con su actividad económica y/o productiva, sino también sometidas en una vulnerabilidad social y económica.

Con lo anterior no pretendo decir que la situación de todos los pastores varones es mejor en condiciones que la de las mujeres. Existen también pastores en estas denominaciones que no están exentos de vivir esta situación; sin embargo, se debe recordar que en la condición genérica de las mujeres también se intersectan otras condiciones de vulnerabilidad que las ponen en una situación posiblemente más adversa.

Respecto al cuidado en la vejez, para las religiosas consagradas es una garantía que la estructura religiosa provee. Cuando las religiosas deciden dejar de trabajar por decisión personal, por condiciones de salud o por cualquier otro motivo que interrumpa la actividad laboral, es la congregación la que asume el cuidado y manutención de sus integrantes en sus respectivas casas congregacionales.

\section{CONCLUSIONES}

A manera de conclusiones, podemos mencionar que el campo religioso y las vivencias que las mujeres experimentan como parte de sus creencias religiosas han sido considerados un aspecto de la vida privada que esto no debe ser mezclado con la vida secular y, por lo tanto, con la ciudadanía de las mujeres. Podría pensarse que el derecho de las mujeres a tener derechos es algo que sólo debe ser visto a partir de la vida pública, aunque debemos recordar que muchas de las violaciones a los derechos humanos de las mujeres (derechos reproductivos, sexuales y a la no violencia) también ocurren en aquellos espacios que por tradición se han considerado privados u ocultos de las miradas, y que como en el caso de la vida religiosa quedan suspendidos en una especie de "limbo" - recordemos que en la tradición católica, el limbo era el espacio en el que los infantes no bautizados permanecían de manera indefinida, sin condenación ni salvación-, es decir, aquel espacio que queda suspendido entre los límites abstractamente establecidos no sólo 
del mundo público y privado, sino también del mundo religioso y la secularidad higiénica que parece ser requerida para el desarrollo de la vida pública. Pareciera que la subjetividad de la vida religiosa requiere ser vivida de manera individual, privada o incluso oculta y son estas características las que no sólo hacen posible que las violaciones al ejercicio de sus derechos en estos ámbitos sean justificadas desde argumentos morales y religiosos, sino también civiles, para que sean excluidas de la participación y la igualdad de derechos dentro de las diferentes Iglesias.

Los ministerios religiosos ordenados y consagrados son sólo una muestra de un tipo de experiencia religiosa de las mujeres dentro de este campo y aunque pudiera considerarse por ello que no es la vivencia de muchas otras en posiciones no jerárquicas o no reconocidas, esto no demerita el trabajo que realizan en la construcción de igualdad a través de nuevas posiciones y luchas al interior del campo religioso. Por otro lado, no debemos caer en falsos supuestos al pensar que por el hecho de que algunas de ellas han logrado ser ordenadas, estamos hablando de una igualdad ganada o de una posición de privilegios, pues las condiciones en las que lo han conseguido o las condiciones en la que desempeñan su labor aún están lejos de ser una muestra de derechos ganados y ejercidos.

La presencia de las mujeres en el campo religiosos desde estos otros lugares, las coloca como agentes claves en la reconfiguración del poder en dicho campo, en algunos casos, con su sola presencia, y en muchos otros, a través del trabajo que realizan y construyen. Sus narraciones dan cuenta de procesos de construcción de igualdad y ejercicio de derechos dentro de un campo que de manera general no está dispuesto a otorgar y reconocer la agencia de las mujeres fuera de un tutelaje religioso. Por otro lado, sus historias, aunque de manera indirecta, también nos hablan del lugar que ocupan las otras mujeres que participan desde los lugares ya conocidos y cuya situación de reconocimiento, representación y distribución de recursos probablemente sea aún más precaria.

De manera más específica, respecto al trabajo pastoral y/o ministerial religioso de las mujeres, podemos mencionar que el desigual valor otorgado a su trabajo, expresado en la diferencia de las condiciones en que lo realizan, nos muestra dos aspectos importantes: el primero, que se trata del producto de una desigualdad centrada en marcos institucionales eclesiales, económicos, sociales e incluso políticos al interior de estas instituciones, en los que detrás de la aparente centralidad de las mujeres en sus familias y actividades de servicio y cuidado, se enmascaran y justifican la exclusión y la no participación en condiciones de paridad. Y segundo, que también es parte de la desigualdad producida por el imaginario social de tipo religioso, que dentro de este campo adquiere un carácter de orden natural y divino que estructura el mundo y el lugar de las personas, no sólo de aquellas que participan del mundo religioso sino de la religiosidad inmersa en la cultura que no sólo divide el mundo, sino que justifica su desigualdad.

Es por todo lo anterior que resulta relevante la mirada a este espacio límbico en el que las 
mujeres están construyendo otras religiosidades desde un ejercicio y una práctica religiosa que las coloca dentro del campo como sujetos y con ello la posibilidad de hacer modificaciones en la balanza del intercambio de saberes y bienes. ¿ 


\section{BIBLIOGRAFÍA}

Abramo, Laís (2004), ¿Inserción laboral de las mujeres en América Latina: una fuerza de trabajo secundaria?, en Revista Estudos Feministas, vol. 12, núm. 2, pp. 224-235.

Bandini, Claudirene (2015), “Ide e pregai: Os desafios sociais de jovens missionárias e suas possibilidades de equidade de gênero no universo religioso", en História, saude e direitos: Sabores e saberes do IV Congresso Latino-Americano de Gênero e Religião, Musskopf \& Blasi Facultadades EST-RSBrasil [en prensa].

Bourdieu, Pierre y Loïc Wacquant (2005), Una invitación a la sociología reflexiva. Buenos Aires, Siglo XXI.

Bourdieu, Pierre (2006), "Génesis y estructura del campo religioso”, en Relaciones. Estudios de Historia y Sociedad, vol. 27, núm. 108, pp. 29-83.

Campos Machado, Maria Das Dores (2005), "Representações e relações de gênero nos grupos pentecostais", en Revista Estudos feministas, vol. 13, núm. 3, pp. 387-396. やDOI: http://dx.doi.org/10.1590/ S0104-026X2005000200012

Campos Machado, Maria das Dores, dos Passos Guimarães, Christiane, Bandeira de Oliveira, Clara, Rabelo, Carla (2006), "La política: un nuevo espacio de articulación de las identidades religiosas y de género", en Si Somos Americanos. Revista de Estudios Transfronterizos, vol. 8, núm. 2, pp. 87-108, URL:_http://www.sisomosamericanos.cl/index.php/sisomosamericanos/article/view/296/257

Castañeda, Patricia (2008), Metodología de la investigación feminista. México, Fundación Guatemala, UNAM, CEIICH.

De la Torre, Renée y Patricia Fortuny (1991), “La mujer en la 'Luz del Mundo’. Participación y representación simbólica”, en Estudios sobre las culturas contemporáneas, vol. 4, núm. 12, pp. 125-150.

De Souza, Rosane Aparecida (2015), "Igrejas Evangélicas fundadas por mulheres na região industrial de contagem, Minas Gerais”, IV Congresso Latino-Americano Gênero e Religião, 5-8 de agosto de 2015, Rio Grande Brasil. 
Fortuny, Patricia (2001), “Religión y figura femenina: entre la norma y la práctica”, Revista de Estudios de Género. La ventana, núm. 14, pp. 126-158.

Fraser, Nancy (2006), "La justicia social en la era de la política de la identidad: redistribución, reconocimiento y participación”, en Nancy Frasser y Axel Honneth, ¿Redistribución o reconocimiento? Un debate político filosófico. Madrid, Morata.

Fraser, Nancy (2008), Escalas de justicia. Barcelona, Herder.

INEGI (2015), Clasificación de religiones 2010 / Instituto Nacional de Estadística y Geografía, Secretaría de Gobernación. México, INEGI, c2015. Consultado el 5 de marzo de 2016, URL: http://internet. contenidos.inegi.org.mx/contenidos/productos/prod_serv/contenidos/espanol/bvinegi/productos/nueva_ estruc/702825064983.pdf

Jelin, Elizabeth (s/f), "Igualdad y Diferencia: Dilemas de la Ciudadanía de las Mujeres en América Latina", en Ágora. Cuadernos de estudios políticos, año. 3, núm. 7, pp. 189-214.

Juárez, Elizabeth (2000), "Re-creando identidades en dos grupos religiosos", en Revista de Estudios de Género. La Ventana, núm. 12, pp. 74-111.

Kawulich, Barbara (2005), "La observación participante como método de recolección de datos", en FQS, Forum Qualitative Sozialforschung / Forum: Qualitative Social Research, vol. 6, núm. 2, art. 43, consultado el 22 de octubre de 2013, URL: http://www.qualitative-research.net/fqs/

Lagarde, Marcela (1990), “Identidad Femenina”, URL: http://xenero.webs.uvigo.es/profesorado/purificacion_ mayobre/identidad.pdf

Lagarde, Marcela (1996), Género y feminismo: desarrollo humano y democracia. Cuadernos inacabados 25. Madrid, Horas y Horas.

Lamas, Marta (2001), "Prólogo", en Celia Amorós, Feminismo: igualdad y diferencia. México, Programa Universitario de Estudios de Género, UNAM, pp. 9-17.

Tamayo, Juan José (2011), Otra teología es posible: pluralismo religioso, interculturalidad y feminismo. España, Herder. 
Vélez, Graciela (2006), “Género y ciudadanía, las mujeres en el proceso de construcción de la ciudadanía”, en Espacios Públicos, vol. 9, núm. 17, UAEM, pp. 376-390.

Fecha de recepción: 18 de noviembre de 2015

Fecha de aceptación: 18 de mayo de 2016

\section{ACERCA DE LA AUTORA}

\section{SANDRA VILLALOBOS NÁJERA}

(savinadh@hotmail.com)

Es licenciada en psicología por la Universidad Autónoma de Aguascalientes y maestra en desarrollo humano por la Universidad Veracruzana. Actualmente es doctorante en la Facultad de Ciencias Políticas y Sociales con orientación en sociología de la Universidad Nacional Autónoma de México (UNAM). Es, también, diplomada en "Feminismo en América Latina", por el Centro de Investigaciones Interdisciplinarias en Ciencias y Humanidades (CEIICH), de la UNAM, y en 2015 realizó una estancia de investigación en la Universidade Federal do Rio de Janeiro en Rio de Janeiro, en Brasil. 